\title{
Visualization of Network Structure in Poly(butadiene)/Polystyrene Pseudo Interpenetrating Polymer Networks by Transmission Electron Microscopy
}

\author{
H. L. Frisch and Yongpeng XuE \\ Department of Chemistry, State University of New York at Albany, \\ Albany, New York 12222, U.S.A.
}

(Received November 24, 1993)

\begin{abstract}
Pseudo interpenetrating polymer networks (PIPNs) of 1,4-cis-poly(butadiene) and polystyrene (PS) have been prepared to directly examine the polymer network structure in IPNs by transmission electron microscopy (TEM). Samples of crosslinked cis-poly(butadiene) (PB) were swollen with styrene monomer, which then was polymerized in situ to form sequential PIPNs. TEM observations were performed using samples stained with $2 \%$ osmium tetroxide following the procedure of Shiibashi et al. Network structures of crosslinked cis-PB were visualized in the PIPNs. The mean size of the unit roughly square plaquette of the network seen in the electron micrographs was in relatively good agreement with the unperturbed root mean square end-to-end distance of PB sub-chains between crosslinks.

KEY WORDS Pseudo Interpenetrating Polymer Networks / Network Stucture / Sub-Chains / End-to-End Distance / Transmission Electron Microscopy / Poly(butadiene) / Polystyrene /
\end{abstract}

Interpenetrating polymer networks (IPNs) (including full IPNs and pseudo IPNs) can be formed in a variety of ways, but in each case it must involve the polymerization or crosslinking of at least one of the component polymers in the presence of the other one. If only one of the polymers in any IPN is crosslinked, the material is referred to as a pseudo IPN (PIPN) or semi-IPN.

Because most of the synthetic IPNs will require a detailed understanding at the network and domain levels, approaches to examine the crosslinked network structure in IPNs should be an important aspect of IPN characterizations. Although conventional electron microscopy (TEM) has been employed previously to measure the domain size and the microphase separation structures of IPNs, TEM generally cannot image single unstained common polymer molecules within solids, liquids, gels or at interfaces. This is due primarily to the inherent lack of contrast and the electron beam damage. But it has been reported by Shiibashi et al. that if the individual polymer molecules in a solution could be fixed unchanged just as they are, we could directly see the structure of the molecules, the gels and the networks of the cured rubber by TEM. ${ }^{1,2}$ Similarly, if the polymer chains in the crosslinked network of an IPN or a PIPN can be fixed as they are, then we could examine directly the network structure of the crosslinked polymers by TEM.

Since morphology plays a major role in affecting IPN properties, it has been extensively studied by electron microscopy and glass transition behavior. IPNs reported can be classified as having large domain structures with sizes around a few microns, ${ }^{3}$ intermediate structures with sizes around $1000 \AA,{ }^{4,5}$ fine structures with sizes around $100 \AA,{ }^{5}$ and no resolvable domain structures at all. ${ }^{6-8}$

The nature of interpenetration is believed to 
vary systematically with the morphology, from partial interpenetration mostly at the boundaries of the dispersed phase (in the cases of large domain structure IPNs), to a supermolecular mixing where the domain size is of the same order of magnitude as the distance between the crosslinks of the polymer network (a domain size of $100 \AA$ corresponds roughly to a distance of about 200 mers between crosslinks), ${ }^{5}$ and to a molecular level mixing (homogeneous IPNs). In this paper we report on the employment of the method in reference 1 and 2 to prepare TEM samples of pseudo IPNs of crosslinked PB and linear PS, and the subsequent investigation of their morphologies via transmission electron microscopy.

\section{EXPERIMENTAL}

\section{Materials}

The raw materials used and their descriptions are listed in Table I. EGDMA was washed with $10 \%$ aqueous sodium hydroxide and then distilled at $35-40^{\circ} \mathrm{C}$ under vacuum of about $2 \mathrm{mmHg}$. BPO was dissolved in carbon tetrachloride, precipitated, washed with cool methanol, and then dried at room temperature under a vacuum of about $2 \mathrm{mmHg}$. Styrene was washed with $10 \%$ aqueous sodium hydroxide to remove inhibitor, followed by washing with

Table I. Materials

\begin{tabular}{lll}
\hline Designation & \multicolumn{1}{c}{ Description } & \multicolumn{1}{c}{ Source } \\
\hline PB & $\begin{array}{l}\text { Poly(butadiene), cis, } \\
\bar{M}_{w}=2 \times 10^{5}-3 \times 10^{5},\end{array}$ & Aldrich Chem. Co. \\
& \multicolumn{1}{c}{${ }^{2} .01 \mathrm{~g} \mathrm{~cm}^{-3}}$, & \\
& \multicolumn{1}{c}{$T_{\mathrm{g}}-102^{\circ} \mathrm{C}}$, & \\
& solubility parameter 6.3 & \\
EGDMA & $\begin{array}{l}\text { Ethylene glycol } \\
\text { dimethacrylate, reagent }\end{array}$ & Aldrich Chem. Co. \\
BPO & Benzoyl peroxide (97\%) & Aldrich Chem. Co. \\
Styrene & Reagent (99\%) & Aldrich Chem. Co. \\
Toluene & Reagent & Fisher Scientific \\
Methanol & Reagent & Fisher Scientific \\
Carbon & Reagent & Fisher Scientific \\
tetrachloride & & \\
\hline
\end{tabular}

distilled water, dried over anhydrous $\mathrm{Na}_{2} \mathrm{SO}_{4}$, and distilled at $40^{\circ} \mathrm{C}$ under dry $\mathrm{N}_{2}$ and vacuum. Linear poly(butadiene) (cis) was purified by dissolving it in toluene and precipitating it with methanol. After filtration it was washed several times with methanol and dried under vacuum at room temperature. All solvents were dried over Linde $4 \mathrm{~A}$ molecular sieves.

\section{Preparation of Crosslinked Poly(butadiene)}

Purified PB ( $3.0 \mathrm{~g})$ was dissolved in $45 \mathrm{ml}$ dry toluene. To the solution was added $0.045 \mathrm{~g} \mathrm{BPO}$ and EGDMA crosslinker. The reaction mixture was heated at $60^{\circ} \mathrm{C}$ for $6 \mathrm{~h}$, then poured into Petri dishes whose bottom was sprayed with a universal release agent and heated at $70^{\circ} \mathrm{C}$ for $24 \mathrm{~h}$ under nitrogen gas stream in a vacuum oven. Then the temperature was raised to $80^{\circ} \mathrm{C}$ for another $24 \mathrm{~h}$ under vacuum. The products were almost colorless transparent solids with an $\bar{M}_{\mathrm{c}}$ (molar mass between crosslinks) of 8000,4000 , and 2000 , respectively as calculated from the employed stoichiometry. A swelling experiment was performed in toluene for the sample whose theoretical $\bar{M}_{\mathrm{c}}$ was 8000 . The experimental $\bar{M}_{\mathrm{c}}$ value was 8,890 . It provided the needed evidence that the $\bar{M}_{\mathrm{c}}$ value obtained from a swelling experiment was in fairly good agreement with the stoichiometric, theoretical $\bar{M}_{\mathrm{c}}$ value.

\section{Preparation of Pseudo IPNS}

The crosslinked PB specimens (original thickness of film of the crosslinked PB was cut into $0.4 \times 1.2 \mathrm{~cm}$ rectangular pieces) were swollen for one day in a large volume of styrene/BPO solution ( $1 \%$ by wt of BPO in the volume of styrene monomer). Styrene monomer in the swollen specimens was polymerized in gelatin capsules which were inert to organic solvents, easy to handle, and inexpensive, at $70^{\circ} \mathrm{C}$ for $24 \mathrm{~h}$. After the polymerization of the styrene monomer the original soft specimens became hard materials and the final products were transparent. The formation of sequential PIPNs was confirmed by carefully weighing the 
specimens before swelling in styrene and after polymerization of the swollen samples. All samples increased to 5 times their original weights. These results showed that the composition of different crosslinking density PIPNs was 20:80 by weight of PB to PS.

All samples for TEM were stained for 2 days in $2 \%$ aqueous osmium tetroxide. They were rinsed and dried. The dried samples were embedded in Spurr epoxy resin. Sections were cut into a thickness about $500-1000 \AA$ by a RMC MT-2C ultramicrotome with glass knives, and then were viewed on a Zeiss 902 High Resolution Transmission Electron Microscope. The magnifications employed are shown in Figures 1, 2, and 4.

\section{RESULTS AND DISCUSSION}

The microstructures of polymers are listed in Table II. The molecular weights between crosslinks of PB sub-chains, denoted by $\bar{M}_{\mathrm{c}}$, were varied by varying the quantity of crosslinking agent. The electron micrographs of three samples are shown in Figure 1. The

Table II. The chemical structure of PB and PS polymers

Polymer Structure

dark portions are poly(butadiene) chains stained with $\mathrm{OsO}_{4}$, and bright parts correspond to the unstained polystyrene. ${ }^{9}$ In order to highlight the structure formed by the stained poly(butadiene) chains in the photograph, the whole dark web of lines was outlined with a fine black ink pen and some separated parts
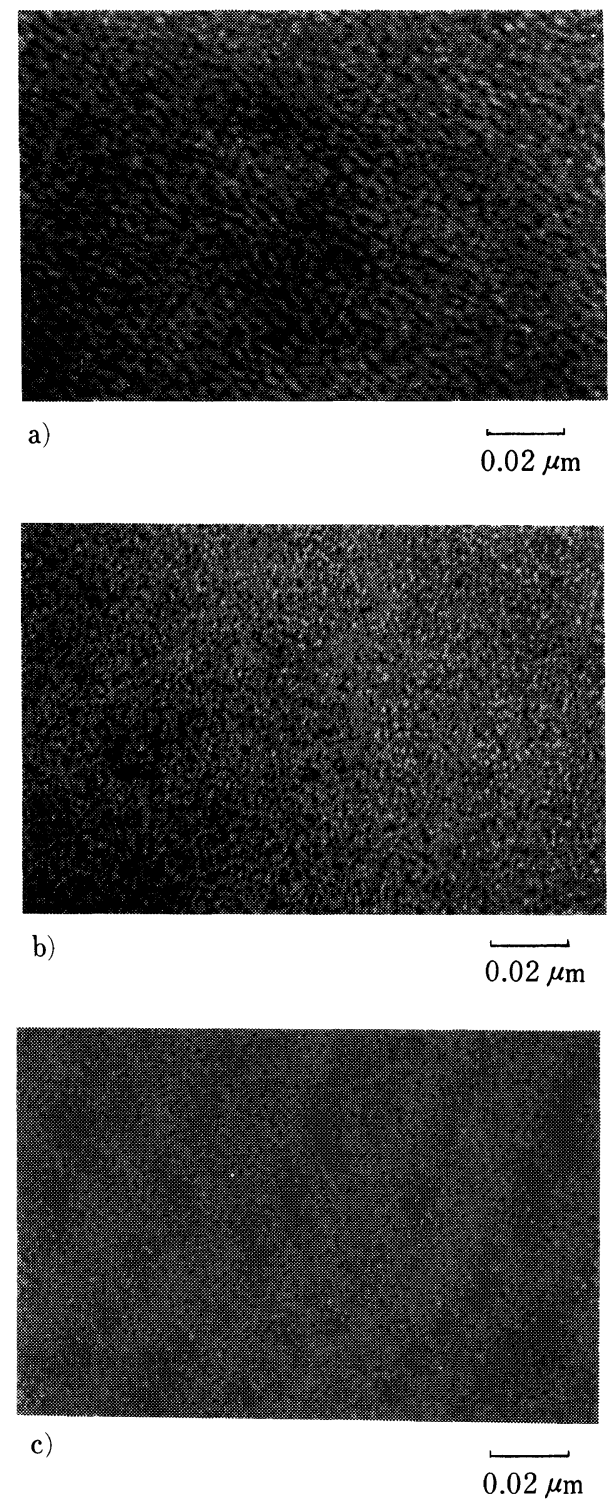

Figure 1. Transmission electron micrographs of CPB/PS PIPNs with various average molecular weights between PB crosslinks. Molecular weight between PB crosslinks $\left(\bar{M}_{\mathrm{c}}\right)$ : (a) $8 \times 10^{3}$; (b) $4 \times 10^{3}$; (c) $2 \times 10^{3}$. 


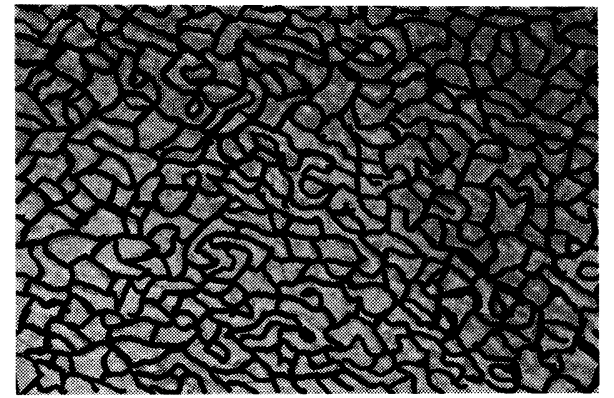

a)

$0.02 \mu \mathrm{m}$

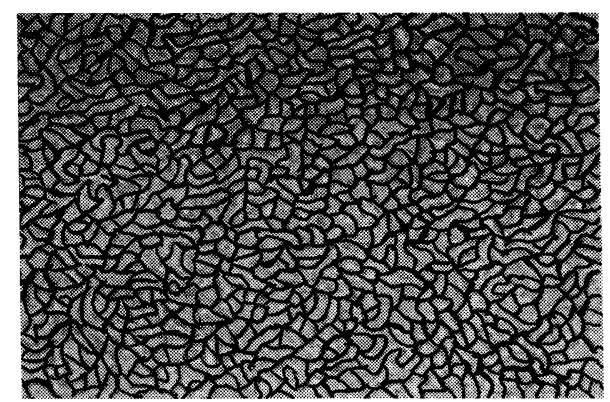

b)

$0.02 \mu \mathrm{m}$

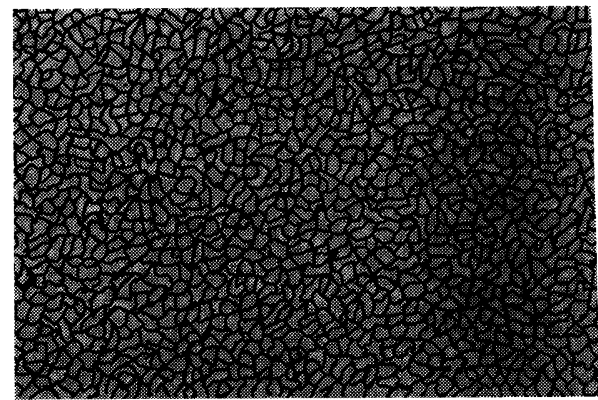

c)

$\widetilde{0.02 \mu \mathrm{m}}$

Figure 2. Schematic network structure of crosslinked PB in PIPNs traced from the electron micrographs in Figure 1. $\bar{M}_{\mathrm{c}}$ of PB crosslinks: (a) $8 \times 10^{3}$; (b) $4 \times 10^{3}$; (c) $2 \times 10^{3}$.

were connected (Figure 2). The average area of the meshes was measured by counting the average number of meshes in known areas of squares. Clearly from such a two dimensional projection the full three dimensional random structure cannot be uniquely reconstructed, yet by changing the average crosslink density

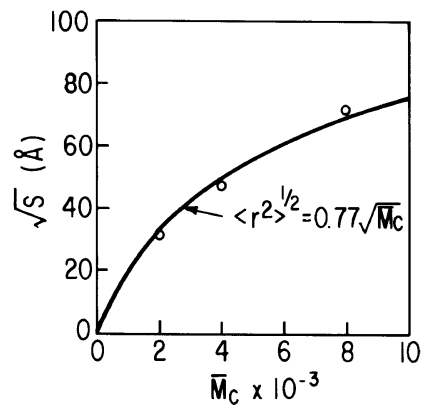

Figure 3. Relation between the root mean square areas of the meshes (on Figure 2) and $\bar{M}_{\mathrm{c}}$ of PB in PIPNs.

chemically of our networks we can check that the change in the apparent cross-sectional area of our tracing, produced as described above, reflects accurately this change in crosslink density. This gives us some confidence in this limited reconstruction.

Figure 3 shows the relation between the root mean square areas of the meshes and $\bar{M}_{\mathrm{c}}$. The root mean square end-to-end distances that correspond to $\bar{M}_{\mathrm{c}}$ were obtained from the equation:

$$
\left\langle r^{2}\right\rangle^{1 / 2}=0.77 \sqrt{\bar{M}_{\mathrm{c}}}
$$

The derivation of this equation was based on the Flory-Fox relation ${ }^{10}$ and the characteristic ratio for $c i s-1,4-\mathrm{PB}$ obtained by Mark. ${ }^{11}$ The distances obtained in this way were in relatively good agreement with the root means sqare areas. These results suggest that the photographs (Figure 1) may reflect the real network structure of the crosslinked poly(butadiene). Electron micrographs of a PIPN with $\bar{M}_{\mathrm{c}}$ of crosslinked PB being equal to 4000 at much higher magnifications show the networks structure more clearly (Figure 4). The average length of the black portions in Figure 4 is about $50 \AA$ which corresponds to the end-to-end distance of PB sub-chains in this sample. It should be pointed out that the electron micrographs presented here represent twodimensional projections of a three-dimensional polymer networks. Therefore the possibility exists that superposition of network structures 


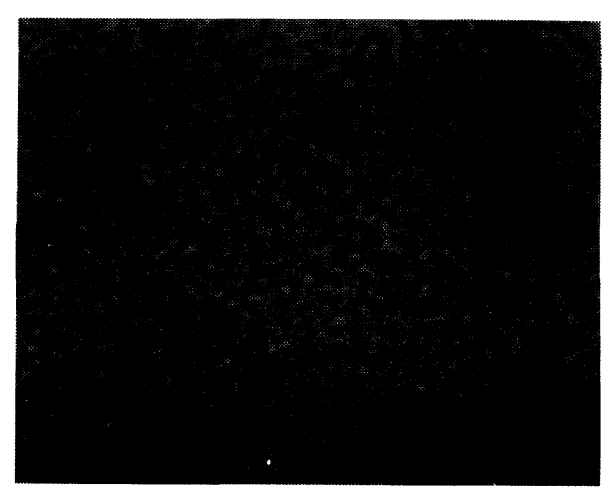

$0.01 \mu \mathrm{m}$

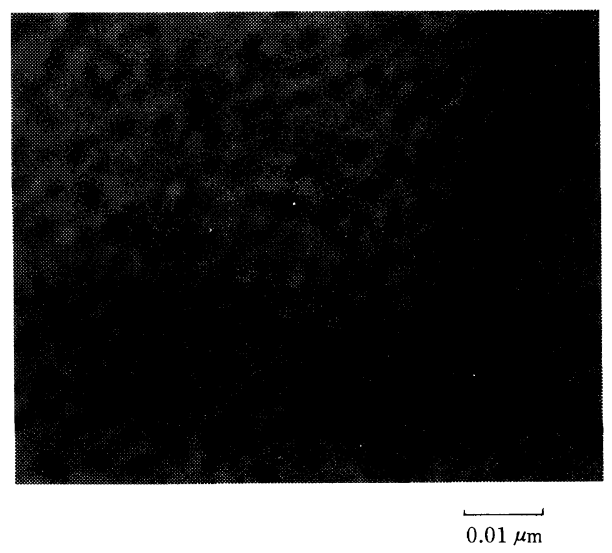

Figure 4. Transmission electron micrographs of PIPN with $\bar{M}_{\mathrm{c}}$ of PB crosslinks being $4 \times 10^{3}$ at higher magnifications.

could influence the apparent mesh size in the micrographs. However, the close agreement between observed and calculated chain dimensions attests to the validity of the method used. Also, the TEM image is not of individual polymer chains, but of supermolecular structures which consist of a number of PB chains. Similar supermolecular structures have been reported in ref 1 and 2 . We expect that this method described for the PB/PS PIPNs could be used in other binary IPN systems if a valid selective stain can be found and no specific interactions between polymer chains exist. Shiibashi et al. described the similar usage in natural rubber/polystyrene and synthetic iso- prene rubber/polystyrene systems. ${ }^{1,2}$

The network structure of crosslinked PB in IPNs could be studied by TEM via a special sample preparation method. The crosslinked PB network structure remains unchanged on swelling with styrene monomer and after styrene polymerization (form PIPNs). The rigid polystyrene macromolecules form closely packed structures in the matrix of crosslinked PB networks. The rigid PS structure strengthens the entire system, playing the role of a hardening filler and allowing easier sectioning for TEM samples. The observed sizes of the crosslinked PB networks are in relatively good agreement with the calculated end-to-end distances that correspond to $\bar{M}_{\mathrm{c}}$.

Acknowledgments. We thank Dr. J. L. Travis for allowing the use of the electron microscopy laboratory at the Biology Department, State University of New York at Albany, U.S.A. This work was supported by the National Science Foundation Grant DMR 9023541.

\section{REFERENCES}

1. T. Shiibashi, K. Hirose, and N. Tagata, Kobunshi Ronbunshu (in Japanese), 46, 465 (1989).

2. T. Shiibashi, K. Hirose, and N. Tagata, Kobunshi Ronbunshu (in Japanese), 46, 473 (1989).

3. D. Klempner, K. C. Frisch, and H. L. Frisch, J. Elastoplastics, 3, 2 (1971).

4. L. H. Sperling, D. A. Thomas, M. J. Covitch, and A. J. Curtius, Polym. Eng. Sci., 12, 101 (1972).

5. L. H. Sperling, D. A. Thomas, and V. Huelck, Macromolecules, 5, 340 (1972).

6. S. C. Kim, D. Klempner, K. C. Frisch, H. L. Frisch, and H. Ghiradella, Polym. Eng. Sci., 15, 39 (1975).

7. K. C. Frisch, D. Klempner, S. Migdal, H. Ghiradella, and H. L. Frisch, Polym. Eng. Sci., 14, 76 (1974).

8. H. L. Frisch and Y.-H. Hua, Macromolecules, 22, 91 (1989).

9. A. E. Woodward, "Atlas of Polymer Morphology," Hanser, New York, N.Y., 1989, Chaptor VI.

10. P. J. Flory and T. G. Fox, J. Am. Chem. Soc., 73, 1904 (1951).

11. J. E. Mark, J. Am. Chem. Soc., 88, 4354 (1966). 